\title{
The predictive role of neutrophil-to-lymphocyte ratio and platelet-to-lymphocyte ratio in children with simple febrile seizures
}

\author{
Meyri Arzu Yoldas ${ }^{1}, \quad$ Fatma Hanci $^{2}, \quad$ Gokce Kaya Dincel $^{\mathbf{1}}$, Mervan Bekdas $^{1}$ \\ ${ }^{I}$ Department of Pediatrics, Bolu Abant Izzet Baysal University, Faculty of Medicine, Bolu, Turkey \\ ${ }^{2}$ Department of Pediatric Neurology, Bolu Abant Izzet Baysal University, Faculty of Medicine, Bolu, Turkey
}

\section{ABSTRACT}

Aim: To evaluate the predictive roles of biochemical and complete blood count parameters in the diagnosis of febrile seizures by comparing these between patients with simple febrile seizures and febrile patients without seizures.

Methods: One hundred fifty-two children (66 girls and 86 boys), aged 6-60 months presenting with fever symptoms presenting to our hospital's pediatric emergency department between January 2015 and January 2020 were included in the study. Demographic data, complete blood count parameters and biochemical parameter levels were compared between the two groups. These were divided into a patient group with simple febrile seizures $(\mathrm{n}=74)$ and a febrile control group without seizures $(\mathrm{n}=$ 78).

Results: Comparison of biochemical parameters revealed significantly higher glucose, CRP, and ALT levels in the febrile seizure group, while $\mathrm{Ca}$ and $\mathrm{Na}$ were significantly lower. Comparison of complete blood count parameters revealed significantly higher white blood cell (WBC), neutrophil, red cell distribution width, neutrophil-to-lymphocyte ratio (NLR), and platelet-to-lymphocyte ratio (PLR) values in the febrile seizure group, while hemoglobin, hematocrit, mean corpuscular volume, lymphocytes, and mean platelet volume were significantly lower.

Conclusions: We think that in addition to markers such as WBC, leukocytes, and CRP for evaluating inflammation in patients with febrile seizures, simple, easily available, and inexpensive tests such as NLR and PLR can also be useful for assessing inflammation.

Key words: Febrile seizure, child, leukocyte count, lymphocytes, platelet count.

$\triangle$ Dr. Meyri Arzu Yoldas

Department of Pediatrics, Bolu Abant Izzet Baysal University, Faculty of Medicine, Bolu, Turkey

E-mail: m.arzuyoldas@gmail.com

Received: 2021-04-08 / Revisions: 2021-04-29

Accepted: 2021-05-04 / Published online: 2021-07-01

\section{Introduction}

Febrile seizure (FS) is seen in $2-5 \%$ of infants and children, and is the most common convulsive event in the pediatric age group. The American Academy of Pediatrics (AAP) defines febrile seizures as those accompanied by fever $\left(>38^{\circ}\right.$ using any measurement technique) in neurologically healthy infants and children (aged between six and 60 months) with no signs of intracranial infection or metabolic disturbance, or history of afebrile seizures. Febrile seizure, the most common form of 
seizure in the pediatric age group, was defined as a seizure associated with febrile illness with no known other cause of acute symptomatic seizure, including such as central nervous system infection or acute electrolyte imbalance [1].

Febrile seizures are also classified as either simple or complex. Complex febrile seizure was defined as either focal or multiple seizures, or seizures with a duration exceeding $15 \mathrm{~min}$, or both. Simple febrile seizure was defined as a single generalized episode occurring within 24 $\mathrm{h}$ and seizures with a duration less than $15 \mathrm{~min}$. Although the etiology of these seizures is still not fully understood, studies have shown the involvement of inflammation [2,3].

Complete blood count parameters currently used in studies for evaluating several conditions, such as acute and chronic inflammatory states, infectious diseases, and malignancies are simple and popular parameters in differential diagnosis. Systemic inflammation and febrile seizure are closely related entities. Various inexpensive and easily accessible laboratory parameters are available for evaluating the severity of systemic inflammation, including the neutrophil: lymphocyte ratio (NLR) and platelet: lymphocyte ratio (PLR). Changes in the proportions of white blood cells (neutrophilia and lymphopenia) represent the immune system's physiological response to an inflammatory process, injury or stress[4], also reflected by NLR and PLR. Some complete blood count parameters have been reported as novel inflammatory markers in Guillain-Barre syndrome and venous thrombosis $[5,6]$.

Studies in recent years have shown that inflammation and hypoxia affect some complete blood count parameters and are associated with seizures [7]. The purpose of this study was to evaluate the predictive roles of biochemical and complete blood count parameters in the diagnosis of febrile seizures by comparing these between patients with simple febrile seizures and febrile patients without seizures.

\section{Materials and methods}

One hundred fifty-two children aged 6-60 months presenting with fever symptoms to our hospital's pediatric emergency department between January 2015 and January 2020 were included in the study. Patients aged between six months and sixty months and meeting the definition of simple febrile seizure were included in the study. Patient data were analyzed retrospectively.

Patients meeting AAP diagnostic criteria were divided into a patient group (=Group 1) with simple febrile seizures $(\mathrm{n}=74)$ and Group 2 with fever but no seizures $(n=78)$. Patients with previous histories of epilepsy or cerebral palsy, neuromotor retardation, chronic illness, head trauma, or diagnosed with meningitis or encephalitis within the previous month or concurrently, were excluded from the study. The data collected included demographics (date of birth, sex), and complete blood count parameters and blood biochemistry values were also compared between the groups.

Complete blood count parameters in blood collected at time of initial presentation included white blood cell (WBC) count, red blood cell (RBC) count, hemoglobin (Hb), mean erythrocyte volume (MCV), hematocrit (HCT), erythrocyte distribution width (RDW), platelet count (PLT), mean platelet volume (MPV), the neutrophil:lymphocyte ratio (NLR), and the platelet:lymphocyte ratio (PLR). NLR was calculated as the ration of lymphocytes to neutrophils, and PLR as that of platelets to lymphocytes (XN-1000-HematologyAnalyzer, Sysmex Corporation, Kobe, Japan). 
Blood biochemistry parameters included sodium $(\mathrm{Na})$, potassium $(\mathrm{K})$, calcium $(\mathrm{Ca})$, aspartate aminotransferase (AST), alanine aminotransferase (ALT), and C-reactive protein (CRP) Automatic Biochemistry Analyzer Abbott Architect c8000, Abbott Laboratories, Abbott Park, IL, USA). The study protocol was approved by the local human research committee (protocol number 2905 2020/179), and the study was conducted in compliance with the Declaration of Helsinki.

\section{Statistical analysis}

Continuous data were expressed as mean \pm SD (min-max) in both study groups, and categorical variables as frequency and percentage. The Kolmogorov-Smirnov test was first applied to assess normality of distribution of the study variables. Normally distributed variables were analyzed using the independent samples t-test, while categorical variables were analyzed using Pearson's chi-square test and Fisher's exact test. The results were assessed within 95\% reliance, with $\mathrm{p}<0.05$ being considered statistically significant. All analyses were performed on Statistical Package for Social Sciences 25.0 for Windows software
(SPSS Inc., Chicago, IL, USA).

\section{Results}

One hundred fifty-two patients were included in the study. Mean ages (months) in group 1 with febrile seizures were 42 months (56.8) among boys and 32 months (43.2) in girls, compared to 44 months (56.4) in boys and 34 months (43.6) in girls in the group $2(\mathrm{p}=0.96)$. Boys constituted 42 (56.8\%) of the group 1, while $34(43.6 \%)$ of the group 2 were girls. No statistically significant difference was determined between the groups in terms of demographic data (Table 1).

In terms of biochemical parameters, CRP, glucose and ALT values were significantly higher in the group 1 than in the group 2, while $\mathrm{Na}$ and $\mathrm{Ca}$ were lower in the group 1 (Table 2). No significant difference was observed between the groups' serum AST and serum K levels.

Comparison of complete blood count parameters revealed significantly higher WBC, neutrophil, RDW, NLR and PLR values in the group 1 compared to the group 2, while $\mathrm{MCV}$, $\mathrm{HB}, \mathrm{HCT}$, and MPV were lower $(\mathrm{p}<0.05)$ (Table 3).

Table 1. Demographic characteristics of the study groups.

\begin{tabular}{|l|c|c|c|}
\hline Parameters & Group 1 (n=74) & Group 2 ( n=78) & p value \\
\hline Age (months), mean + SD & $41,64 \pm 18$ & $36.2 \pm 19,9$ & $0.078^{a}$ \\
\hline $\begin{array}{c}\text { Gender, n (\%) } \\
\text { Male } \\
\text { Female }\end{array}$ & $42(56.8)$ & $44(56.4)$ & $0.96^{b}$ \\
\cline { 2 - 4 } & $32(43.2)$ & $34(43.6)$ & \\
\hline
\end{tabular}

SD: Standard deviation. Data expressed as n (\%). p values indicate statistical significance at 0.05.

${ }^{a}$ Independent samples t-test, ${ }^{b}$ Pearson's chi-square test. 
Table 2. Biochemical parameters of the study groups

\begin{tabular}{|l|c|c|c|}
\hline Parameters & Group 1 $(\boldsymbol{n = 7 4 )}$ & Group 2 $(\boldsymbol{n}=\mathbf{7 8})$ & p value \\
\hline CRP, mg/L & $10.8(0.1-68.7)$ & $4.5(0.1-49.6)$ & $0.003^{a}$ \\
\hline Calcium, mg/L & $9.5(8.3-10.8)$ & $9.8(8.8-10.8)$ & $0.01^{a}$ \\
\hline AST, U/L & $37.5(11-160)$ & $32.7(19-58)$ & $0.1^{a}$ \\
\hline ALT, U/L & $20.3(7-100)$ & $16.4(10-33)$ & $0.006^{a}$ \\
\hline Serum glucose, mg/L & $92.9(38-174)$ & $85.8(63-127)$ & $0.007^{a}$ \\
\hline Sodium, mmol/L & $137.1(131-145)$ & $138.8(134-144)$ & $0.011^{a}$ \\
\hline Potassium, mmol/L & $4.47(3.5-5.5)$ & $4.49(3.8-5.5)$ & $0.78^{a}$ \\
\hline
\end{tabular}

AST aspartate aminotransferase, ALT alanine aminotransferase, CRP C-reactive protein, SD standard deviation. Data expressed as mean (min-max). Bold $p$ values indicate statistical significance at 0.05. ${ }^{a}$ Independent samples t-test.

Table 3. Comparison of hematological parameters in the two groups.

\begin{tabular}{|l|c|c|c|}
\hline Parameters & Group 1 $(\boldsymbol{n}=\mathbf{7 4})$ & Group 2 $(\boldsymbol{n}=\mathbf{7 8})$ & $\boldsymbol{p}$ value \\
\hline WBC, $\times 10^{9} / \mathrm{L}$ & $9.9(4-27.3)$ & $8.6(4.2-16.9)$ & $\mathbf{0 . 0 2 2}^{\mathbf{a}}$ \\
\hline Neutrophil, $\times 10^{9} / \mathrm{L}$ & $6.7(0.7-7.8)$ & $2.8(0.4-7.8)$ & $\mathbf{0 . 0 0 3}^{\mathbf{a}}$ \\
\hline Lymphocyte, $\times 10^{9} / \mathrm{L}$ & $3.7(0.7-11.3)$ & $4.7(0.8-10.8)$ & $\mathbf{0 . 0 0 7}^{\mathbf{a}}$ \\
\hline PLT, $\times 10^{9} / \mathrm{L}$ & $323.3(171-578)$ & $349.2(291-575)$ & $0.08^{\mathrm{a}}$ \\
\hline Hemoglobin,, $\mathrm{g} / \mathrm{L}$ & $11.7(8.5-14.9)$ & $12.4(10.2-14.7)$ & $\mathbf{0 . 0 4 5}^{\mathbf{a}}$ \\
\hline Hematocrit & $35.2 \pm 3.38$ & $37.5 \pm 2.5$ & $\mathbf{0 . 0 2}^{\mathbf{a}}$ \\
\hline MCV & $76.9 \pm 6.6$ & $78.9 \pm 5.5$ & $\mathbf{0 . 0 4 7}^{\mathbf{a}}$ \\
\hline RDW & $16.8 \pm 2.02$ & $13.4 \pm 1.7$ & $\mathbf{0 . 0 4}^{\mathbf{a}}$ \\
\hline MPV & $7.16 \pm 3.6$ & $9.3 \pm 1.2$ & $\mathbf{0 . 0 3 9}^{\mathbf{a}}$ \\
\hline NLR & $3.35 \pm 8.1$ & $0.77 \pm 0.67$ & $\mathbf{0 . 0 0 8}^{\mathbf{a}}$ \\
\hline PLR & $119.9 \pm 83.8$ & $88.1 \pm 45.9$ & $\mathbf{0 . 0 0 5}^{\mathbf{a}}$ \\
\hline
\end{tabular}

WBC white blood cell, NLR neutrophil to lymphocyte ratio, PLR platelet to lymphocyte ratio, SD standard deviation, MPV mean platelet volume, PLT platelet count, RDW red cell distribution width, MCV mean corpuscular volume, MPV mean platelet volume, Data expressed as mean(min-max) or mean $\pm S D$. Bold $p$ values indicate statistical significance at $0.05 .{ }^{a}$ Independent samples t-test.

\section{Discussion}

The laboratory parameters of CRP, ALT, and glucose values were significantly higher in the febrile seizure group in the present study, while $\mathrm{Na}$ and $\mathrm{Ca}$ values were lower. In terms of complete blood count parameters, WBC, neutrophil, RDW, NLR and PLR values were significantly higher in the simple febrile seizure group, while lymphocytes, MCV, HB, HCT, and MPV were lower. Inflammatory cells and 
proinflammatory cytokines are known to play an important role in the etiopathogenesis of febrile seizure. Inflammation is thought to lower the seizure threshold and to increase stimulability in the brain [8].

CRP is an acute phase reactant and a highly important marker associated with inflammation levels independently of age, sex, and physical factors [9]. Yiğit et al. compared CRP levels in children with simple and complex febrile seizures and reported higher levels in patients with complex seizures [8]. Additionally, Romanowska et al. reported higher CRP levels in a febrile patient group without seizures compared with a group with febrile seizures [10]. CRP levels were also significantly higher in the simple febrile seizure group in the present study. The higher CRP in the febrile seizure group in the present research may indicate a role of inflammation in the etiopathogenesis of febrile seizure.

$\mathrm{Na}$ and $\mathrm{Ca}$ electrolyte and blood sugar level irregularities are known to play an important role in the etiology of seizures. Acute symptomatic seizures may be seen in children in case of $\mathrm{Na}$ levels lower than 115 to 120 $\mathrm{mmol} / \mathrm{L}$ or higher than $145 \mathrm{mmol} / \mathrm{L}$. It has been suggested that hyponatremia can lower the seizure threshold in febrile seizures [11]. In their comparison between children with febrile and afebrile acute gastroenteritis-related seizures, Wu YZ et al. reported lower Na levels in the afebrile group [12]. Zifman et al. reported lower $\mathrm{Na}$ values in a febrile patient group in children with acute gastroenteritis-related seizures [13]. In the present study, serum $\mathrm{Na}$ levels were significantly lower in the febrile seizure group. However, the focus of infection in our groups was upper respiratory tract infection.

$\mathrm{Ca}$ levels are also important in the seizure etiopathogenesis. Paresthesia and muscle cramps can be seen in mild hypocalcemia, while a Ca level below $5 \mathrm{mg} / \mathrm{dl}$ is reported to cause seizures [14]. Yousefichaijan $\mathrm{P}$ et al. examined 549 cases of simple febrile seizure and observed low $\mathrm{Ca}$ levels in a small number of patients [15]. Serum Ca levels in the present study were significantly lower in the simple febrile seizure group compared to the febrile illness without seizure group.

AST is abundantly expressed in non-hepatic tissue, while ALT concentrations in nonhepatic tissues are low. High hepatic transaminase levels may represent not only liver damage, but also inflammation, fever, or non-specific tissue damage. $\mathrm{Wu} \mathrm{YZ}$ et al. reported higher AST and ALT in a patient group with febrile seizure [12]. No significant difference was observed in AST levels in the present study, but ALT levels were significantly higher in the febrile seizure group. In contrast, blood sugar levels below $40 \mathrm{mg} / \mathrm{dl}$ or above $400 \mathrm{mg} / \mathrm{dl}$ are thought to cause neuronal over-stimulation and to trigger seizures [14]. Costea et al. compared serum glucose levels in patients with febrile seizure, and reported four-fold higher levels in patients with complex febrile seizure [16]. Similarly in the present study, glucose levels were higher in the simple febrile seizure group compared to the febrile illness without seizure group. Febrile seizures may be one of the risk factors for stress hyperglycemia in children, and further studies are now needed on this subject.

Iron deficiency is known to play a role in the development of febrile seizures [17]. The relationship between iron deficiency anemia and febrile seizure is still the subject of investigation. A Sherjil et al. reported that iron deficiency anemia increased the risk of febrile seizure two-fold [18]. Iron concentrations in blood could not be analyzed in the present study since the test was not routinely requested. One 
previous study comparing types of febrile seizure determined statistically significantly lower $\mathrm{Hb}$, Htc and MCV values in patients with complex febrile seizure [19]. Similarly in the present study, we also observed significantly lower $\mathrm{Hb}$, Htc and MCV values in the febrile convulsion group. In addition, RDW is employed for evaluating the etiology of anemia, exhibits positive correlation with inflammation in some diseases, and is thought to be a potential inflammatory marker [20]. Türay et al observed significantly higher RDW in febrile seizure recurrence [21]. Örnek et al. observed higher mean RDW in their complex febrile seizure group, but the difference was not statistically significant [19]. RDW was significantly higher in the simple febrile seizure group in the present study.

Previous studies have investigated the relationship between MPV, reflecting the proportion and size of platelets produced from bone marrow, and severity of inflammation in various diseases [22]. One study comparing children with febrile seizures reported significantly increased MPV values in a complex febrile seizure group [23], while another study observed no significant difference [24]. MPV values in the present study were lower in the simple febrile seizure group.

Leukocyte, neutrophil, and lymphocyte counts, and the ratios thereof, are an important parameter that can be easily used to show the severity of disease in systemic inflammatory conditions [25]. Gontko-Romanowska et al. compared patients with febrile seizures and febrile patients without seizures and reported significantly higher neutrophil levels and significantly lower lymphocyte levels in their febrile seizure group [10]. Similarly in the present study, neutrophil levels were significantly higher in the febrile seizure group, while lymphocyte rates were significantly lower. Yousefichaijan et al. reported significantly higher leukocyte counts in febrile children compared to children with febrile seizures [15]. Leukocyte counts were also significantly higher in the febrile seizure group in the present study.

NLR and PLR have recently been recognized as markers of systemic inflammation, and are used as predictive indicators in some diseases [4]. Studies have reported significantly higher NLR values in complex febrile seizure groups among patients with febrile seizures [8,26]. Another study reported significantly elevated NLR and PLR values [19]. NLR and PLR values were also significantly higher in the simple febrile seizure group in the present study.

The principal limitations of the present study are its retrospective nature, the low patient number, and the fact that only patients with upper respiratory tract infections were included. Further prospective studies with larger patient numbers are now needed.

\section{Conclusion}

Inflammation marker elevation must be carefully considered in patients with febrile seizures. We think that markers such as leukocyte count and CRP are simple, readily available and inexpensive tests. NLR and PLR values can also be employed as predictive indicators.

Funding: The author(s) received no financial support for the research, authorship, and/or publication of this article.

Conflict of Interest: The authors declare that they have no conflict of interest.

Ethical statement: The study was approved by the Local Ethics Committee of Bolu Abant Izzet Baysal University (Date and Decision no: 2020-05-12/112), and written informed consent was obtained from each subject. 


\section{Open Access Statement}

This is an open access journal which means that all content is freely available without charge to the user or his/her institution under the terms of the Creative Commons Attribution NonCommercial License

(http://creativecommons.org/licenses/bync/4.0). Users are allowed to read, download, copy, distribute, print, search, or link to the full texts of the articles, without asking prior permission from the publisher or the author.

\section{References}

[1]Subcommittee on Febrile Seizures; American Academy of Pediatrics. Neurodiagnostic evaluation of the child with a simple febrile seizure. Pediatrics. 2011;127(2):389-94.

[2]Güneş M, Büyükgöl H. Relationship between generalized epileptic seizure and neutrophil/lymphocyte ratio, platelet/lymphocyte ratio, and neutrophil mediated inflammation. Int $\mathrm{J}$ Neurosci. 2020; 130(11): 1095-100.

[3]Aronica E, Bauer S, Bozzi Y, et al. Neuroinflammatory targets and treatments for epilepsy validated in experimental models. Epilepsia. 2017; 58 (Suppl 3): 2738 .

[4]Zahorec R. Ratio of neutrophil to lymphocyte counts--rapid and simple parameter of systemic inflammation and stress in critically ill. Bratisl Lek Listy. 2001; 102(1): 5-14.

[5]Ozdemir HH. Analysis of the albumin level, neutrophil-lymphocyte ratio, and plateletlymphocyte ratio in Guillain-Barré syndrome. Arq Neuropsiquiatr. 2016; 74(9): 718-22.

[6]Artoni A, Abbattista M, Bucciarelli P, et al. Platelet to Lymphocyte Ratio and Neutrophil to Lymphocyte Ratio as Risk Factors for
Venous Thrombosis. Clin Appl Thromb Hemost. 2018; 24(5): 808-14.

[7]Eroglu T, Aydin Turkoglu S, Bolac ES, et al. Hemogram parameters in epilepsy may be indicators of chronic inflammation and hypoxemia. J Neurol Clin Neurosci. 2017; 1(1): 17-20.

[8]Yigit Y, Y1lmaz S, Akdoğan A, et al. The role of neutrophil-lymphocyte ratio and red blood cell distribution width in the classification of febrile seizures. Eur Rev Med Pharmacol Sci. 2017; 21(3): 554-59.

[9]Bilgir O, Bilgir F, Calan $\mathrm{M}$, et al. Comparison of pre- and post-levothyroxine high-sensitivity c-reactive protein and fetuin-a levels in subclinical hypothyroidism. Clinics (Sao Paulo). 2015; 70(2): 97-101.

[10] Gontko-Romanowska K, Żaba Z, Panieński $P$, et al. The assessment of laboratory parameters in children with fever and febrile seizures. Brain Behav. 2017; 7(7): e00720.

[11] Kiviranta T, Airaksinen EM. Low sodium levels in serum are associated with subsequent febrile seizures. Acta Paediatr. 1995; 84(12): 1372-74.

[12] Wu YZ, Liu YH, Tseng CM, et al. Comparison of Clinical Characteristics Between Febrile and Afebrile Seizures Associated With Acute Gastroenteritis in Childhood. Front Pediatr. 2020; 8: 167.

[13]Zifman E, Alehan F, Menascu S, et al. Clinical characterization of gastroenteritisrelated seizures in children: impact of fever and serum sodium levels. J Child Neurol. 2011; 26(11): 1397-1400.

[14] Karceski S. Acute symptomatic seizures and systemic illness. Continuum (Minneap Minn). Neurology of Systemic Disease. 2014; 20 (3 ): 614-23.

[15] Yousefichaijan P, Dorreh F, Abbasian L, et al. Assessing the prevalence distribution of 
abnormal laboratory tests in patients with simple febrile seizure. J Pediatr Neurosci. 2015; 10(2): 93-97.

[16] Costea RM, Maniu I, Dobrota L, et al. Stress Hyperglycemia as Predictive Factor of Recurrence in Children with Febrile Seizures. Brain Sci. 2020; 10(3): 131.

[17]Akbayram S, Cemek M, Büyükben A, et al. Major and minor bio-element status in children with febrile seizure [published correction appears in Bratisl Lek Listy. 2012; 113(9): 572.

[18] Sherjil A, Saeed Z, Shehzad S, et al. Iron deficiency anaemia-a risk factor for febrile seizures in children. J Ayub Med Coll Abbottabad. 2010; 22(3): 71-73.

[19] Örnek Z, Kardeş H, Pişkin İE, et al. Comparison of hemogram parameters in febrile seizures types. Duzce Med J. 2020; 22(1): 1-6.

[20]Lippi G, Targher G, Montagnana M, et al. Relation between red blood cell distribution width and inflammatory biomarkers in a large cohort of unselected outpatients. Arch Pathol Lab Med. 2009; 133(4): 628-32.

[21]Turay, S., Hanci, F., \& Ozde, S. (2021). An overview of vitamin B12 and iron deficiencies as a risk factors in children with febrile seizure etiology. Exp Biomed Res. 2021; 4(2): 154-63.

[22] Yıldız Y, Cakmak S, Calapoğlu T, et al. Mean platelet volume can be used as a hospitalization criteria in pediatric patients diagnosed with acute bronchiolitis. Acta Medica Mediterranea. 2018; 34(6): 19972000.

[23] Özkale M, Erol İ, Özkale Y, et al. Association between platelet indices and febrile seizures in children. Cukurova Med J. 2016; 41(4): 695-701.

[24]Nikkhah A, Salehiomran MR, Asefi SS. Differences in mean platelet volume and platelet count between children with simple and complex febrile seizures. Iran J Child Neurol. 2017; 11(2): 44-47.

[25]Zahorec R. Ratio of neutrophil to lymphocyte counts-- rapid and simple parameter of systemic inflammation and stress in critically ill. Bratisl Lek Listy. 2001; 102(1): 5-14.

[26]Liu Z, Li X, Zhang M, et al. The role of Mean Platelet Volume/platelet count Ratio and Neutrophil to Lymphocyte Ratio on the risk of Febrile Seizure. Sci Rep. 2018; 8(1): 15123. 